\title{
Rapid and Accurate Identification of Adulterants via an Electronic Nose and DNA Identification Platform: Identification of Fake Velvet Antlers as an Example
}

\author{
Guojie Xu, Chunsheng Liu, Caili Liao, Xiaolei Ren, \\ Xinyue Zhang, Xiaorui Fu, and Xueyong Wang \\ School of Chinese Materia Medica, Beijing University of Chinese Medicine, No. 6, Wang Jing Zhong Huan South Street, \\ Chaoyang District, Beijing 100102, China
}

Correspondence should be addressed to Chunsheng Liu; max_liucs@263.net and Xueyong Wang; wxyph.d@163.com

Received 19 June 2015; Revised 26 October 2015; Accepted 27 October 2015

Academic Editor: Kourosh Kalantar-Zadeh

Copyright (C) 2016 Guojie Xu et al. This is an open access article distributed under the Creative Commons Attribution License, which permits unrestricted use, distribution, and reproduction in any medium, provided the original work is properly cited.

Background. Adulterants in Chinese medicines had always affected the efficacy of Chinese medicines and resulted in safety problems in drug use. We aimed to take the identification of fake velvet antlers as an example for establishment of a rapid and accurate identification platform for modern pharmaceutical companies and markets of Chinese medicine. Methods. In this study, we developed a novel electronic nose and DNA identification platform for identifying fake velvet antlers. Electronic nose was designed for rapid screening, while accurate identification based on DNA method was performed when electronic nose fails to accurately identify some complex samples. Results. Rapid identification of different velvet antlers based on electronic nose showed the good identification power, while accurate identification based on DNA sequencing technology demonstrated a correct identification rate of $100 \%$. We also mined thirty conserved specific sites of fake velvet antlers, which was useful for further work. Conclusions. The results suggested that electronic nose and DNA identification platform was effective for rapid and accurate identification of fake velvet antlers. It highly improved efficiency and decreased cost of identification and could provide a new sight and inspiration for identification in the field of traditional Chinese medicine (TCM).

\section{Introduction}

Adulterants in Chinese medicines had always affected the efficacy of Chinese medicines and resulted in safety problems in drug use. However, modern pharmaceutical companies and markets of Chinese medicine do not have a suitable platform for rapid and accurate identification of adulterants. Velvet antler (Lu-Rong in China), for instance, the nonossific horn of Cervus nippon or Cervus elaphus, is a precious animalsourced Chinese medicine in China. Pharmacological studies had proven its functions of anti-bone resorption [1], antiarthritis [2], and promoting chondrocyte proliferation [3]. Unfortunately, official velvet antlers are always replaced by the fake velvet antler of Rangifer tarandus due to high profit in Chinese market [4-6]. It is, therefore, necessary to develop a rapid and accurate identification platform for identification of adulterants.
DNA sequencing technology which can mirror abundant DNA information is good candidate for accurate identification of adulterants [4-10]. DNA sequences can be directly determined by fluorescence sensors of DNA sequencer. However, it is also always of low efficiency and high cost. Electronic nose is an intelligent design of simulating human's olfactory system. Its array of metal oxide sensors interacting with a broad range of chemicals with varying intensities is used to rapidly describe the substances of odors. Compared with conventional DNA identification, electronic nose has high efficiency and low cost for identification.

It can be imaged that DNA sequencing method, combined with electronic nose, can highly improve efficiency and decrease cost of identification. Here, we introduced electronic nose into the discrimination of traditional Chinese medicines (TCM) and further developed a novel electronic nose and DNA identification platform for identification of fake velvet 


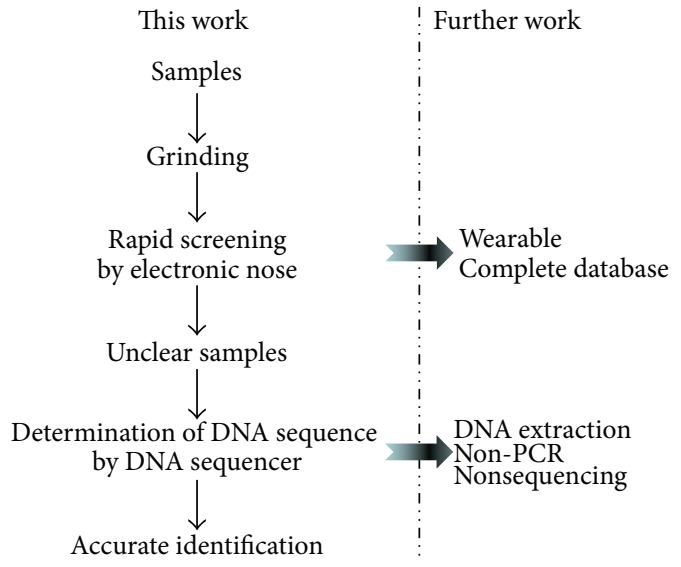

Figure 1: Instruction of electronic nose and DNA identification platform.

TABLE 1: Details of the samples.

\begin{tabular}{lll}
\hline $\begin{array}{l}\text { Velvet } \\
\text { antlers }\end{array}$ & Source & $\begin{array}{l}\text { Assession number of DNA sequences in } \\
\text { Genbank }\end{array}$ \\
\hline & Sequences of current study: \\
& KJ205534-KJ205542 \\
& Sequences of previous study: \\
& EU835711-EU835715 \\
& GQ329010-GQ329015 \\
Authentic & & JF700148-JF700150 \\
& & Sequences of current study: \\
& KJ205543-KJ205557 \\
& Sequences of previous study: \\
& EU835707-EU835709 \\
& & JF443209-JF443224, JF700147 \\
& GQ329007-GQ329009 \\
\hline & Sequences of current study: \\
Fake & KJ205565-KJ205580 \\
& R. tarandus & Sequences of previous study: \\
& JF443384-JF443512 \\
\hline
\end{tabular}

antlers (Figure 1). We aimed to take the identification of fake velvet antlers as an example for establishment of a rapid and accurate identification platform for modern pharmaceutical companies and markets of Chinese medicine, which is of great significance to standardization, normalization, and medication safety of Chinese medicine.

\section{Material and Methods}

2.1. Experimental Materials. The samples were collected from different medicinal velvet antlers markets in China identified by Professor Chen Daixian of Dalian Institute for Drug Control according to the features of the pharmacopoeia of China and previous work [11, 12]. All the samples were deposited into the specimen room of Beijing University of Chinese Medicine. Details of the samples were listed in Table 1.

2.2. Electronic Nose. A FOX-3000 (Alpha MOS, Toulouse, France), which consisted of a sampling apparatus, array of
TABLE 2: The components and main application of sensors of the FOX-3000 electronic nose.

\begin{tabular}{lcl}
\hline Number & Name & Main application \\
\hline S1 & LY2/LG & Oxidizing gas \\
S2 & LY2/G & Ammonia, carbon monoxide \\
S3 & LY2/AA & Ethanol \\
S4 & LY2/GH & Ammonia/organic amine \\
S5 & LY2/gCTL & Hydrogen sulfide \\
S6 & LY2/gCT & Propane/butane \\
S7 & T30/1 & Organic solvents \\
S8 & P10/1 & Hydrocarbons \\
S9 & P10/2 & Methane \\
S10 & P10/2 & Fluorine \\
S11 & T70/2 & Aromatic compounds \\
S12 & PA/2 & Ethanol, ammonia/organic amine \\
\hline
\end{tabular}

sensors, HS-100 autosampler, air generator equipment, and software (Alpha Soft V11) for data recording and analyzing, was used. The sensor array was composed of 12 metal oxide sensors divided into three chambers: T, P, and LY. These metal oxide sensors could catch the electrical signals from redox reaction with gaseous chemical compounds. The components and main application of sensors of the FOX-3000 electronic nose were listed in Table 2. The response values of samples of fake velvet antlers were recorded according to the previous work [13]. The data sets of official or authentic velvet antlers also referred to our previous work [13].

2.3. Statistical Processing of Electronic Nose. Each sample was repeated three times. 10 response values at a period of time $(5 \mathrm{~s}, 10 \mathrm{~s}, 15 \mathrm{~s}, 20 \mathrm{~s}, 25 \mathrm{~s}, 30 \mathrm{~s}, 35 \mathrm{~s}, 40 \mathrm{~s}, 45 \mathrm{~s}$, and $50 \mathrm{~s})$ of each sensor were collected. Raw response values of electronic nose were primary-processed artificially. Some values with no response to all samples were deleted. The resulting data sets of samples were analyzed by SPSS 19.0 based on linear discriminant analysis (LDA).

2.4. DNA Extraction and PCR Amplification. Partial cytochrome oxidase subunit I (COI) gene of $618 \mathrm{bp}$ was used for discrimination of different velvet antlers. DNA was extracted using a common animal DNA extraction kit (Biological Technology Co. Ltd., China). The primers for amplification of partial COI genes were CF (5'-CGGTACTCTGTATCTACTATTTGGTG- $\left.3^{\prime}\right)$ and CR ( $5^{\prime}$-GGGTGACCAAAGAATCAGAATAAGTG-3 ${ }^{\prime}$ ). PCR was performed in a $50 \mu \mathrm{L}$ reaction volume, containing $10 \mathrm{x}$ PCR Buffer $(5.0 \mu \mathrm{L})$, dNTPs (0.4 mM each), $\mathrm{MgCl}_{2}(3.0 \mathrm{mM}), \mathrm{CF}(0.2 \mu \mathrm{M})$, CR $(0.2 \mu \mathrm{M})$, template DNA $(240 \mathrm{ng})$, and Taq polymerase $(0.25 \mathrm{U})$. The amplification profile consisted of $5 \mathrm{~min}$ at $94^{\circ} \mathrm{C}, 35$ cycles of $30 \mathrm{~s}$ at $95^{\circ} \mathrm{C}, 30 \mathrm{~s}$ at $50^{\circ} \mathrm{C}, 60 \mathrm{~s}$ at $72^{\circ} \mathrm{C}$, and an additional extension for $10 \mathrm{~min}$ at $72^{\circ} \mathrm{C}$. The sequences of the PCR products were determined by 3730xl DNA Analyzer (Shanghai Sangon Biological Technology Co. Ltd., China).

2.5. DNA Analysis. Eighty DNA sequences of forty samples were determined by DNA Analyzer. All the sequences were 


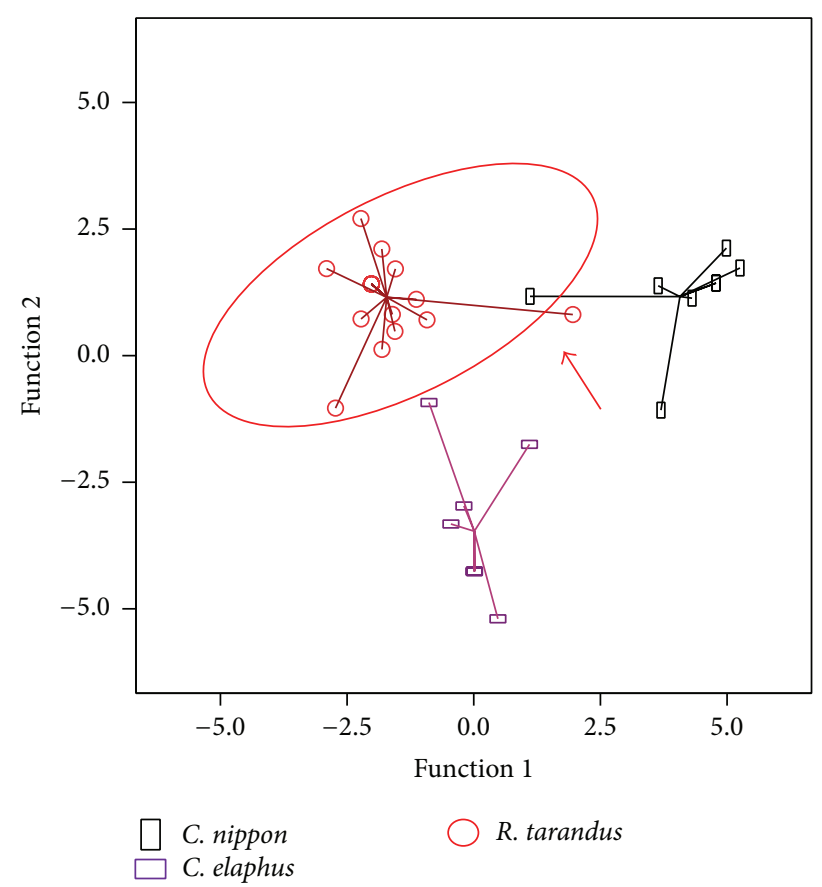

FIGURE 2: Data analysis of electronic nose based on LDA model.

spliced by free ContigExpress software. Sequences were then aligned by clustalW through MEGA 5.0. Specific sites for fake velvet antlers were determined at last and arranged by Excel software. Phylogenetic trees were constructed by NeighborJoining methods using Kimura's 2-parameter model with bootstrap testing of 1000 replicates through MEGA 5.0 [14]. 166 related sequences previously registered in Genbank database (http://www.ncbi.nlm.nih.gov/) were collected for further verifying discriminative power of COI sequences and conservation of specific sites. The analysis method was the same as procedure mentioned above.

\section{Results and Discussion}

3.1. Rapid Discrimination Based on Electronic Nose. Velvet antlers are animal-sourced medicinal materials. Actually, rare studies had reported about discrimination of animalsourced medicinal materials based on specific smells. We firstly established the electronic nose to assess the quality of different deer antler slices [13], and this study is the first attempt to test the electronic nose for discrimination of fake animal-sourced medicinal species.

We previously had understood that LDA model was suitable for modeling of velvet antlers [13], so LDA directly was used to test their applicability of discriminating fake velvet antlers in this study. As a result, most samples were successfully discriminated based on LDA model (Figure 2). It could be implied that electronic nose based on LDA model was effective for rapid identification of velvet antlers. Results also indicated that good response values to velvet antlers were collected from S6 to S12 (data not shown), which were used as important distinguishing sensors for fake velvet antlers.
However, we also found that a few samples, which were highlighted by red arrows in Figure 2, were not well discriminated. To explore the reasons that affect the discrimination power of electronic nose, we classified the samples into four presentations including wax slices, powder slices, sand slices, and bone slices, because we deduced that distinctive smells from different presentations may cover up the species-specific smells. Results showed that smells of sand slices and bone slices were distinguishable, but a few samples of wax slices and powder slices were undistinguishable (Figure 3 ). This indicated that smells of wax slices and powder slices may affect the species-specific smells of samples.

Traditionally, identification of Chinese medicines always depends on appearance and microscopic features of samples in enterprises and markets of Chinese medicine. These kinds of traditional methods are of high subjectivity and always rely on experience of identifier. In comparison to other methods, electronic nose is more simple and objective. But some problems of electronic nose should also be addressed in the future. First, the use of electronic nose in the field of traditional Chinese medicine (TCM) is still in the beginning stages; the establishment of big electronic nose database of different Chinese medicines is necessary for rapid identification. Second, Chinese medicines are always processed by various methods; thus the effects of different processing methods on smells should be taken into consideration. Third, in order to meet the need of identifiers, proper identification model should be built and portable electronic nose drive should be developed for specific Chinese medicines. Last but not least, other methods such as DNA identification method should be in federated manner applied to assure the identification power when some other smells confused detection of electronic nose.

3.2. Accurate Identification Based on DNA Sequencing. Partial COI gene, about $600 \mathrm{bp}$, had been acknowledged as universal DNA identification sequence for discrimination of animal species [4]. Velvet antlers are parts of animal bodies. Therefore, it is feasible to use partial COI sequences for discrimination of different velvet antlers in this study.

Specific sites of sequences of fake velvet antlers were screened by MEGA 5.0. Thirty conserved specific sites of fake velvet antlers were finally mined in total, including site 3 , site 21 , site 27 , site 45 , site 48 , site 54 , site 78 , site 81 , site 156 , site 198 , site 237 , site 238 , site 285 , site 300 , site 304 , site 330 , site 385 , site 393 , site 429 , site 435 , site 444 , site 457 , site 465 , site 471 , site 474 , site 478 , site 493 , site 507 , site 552 , and site 570 (Figure 4). These sites were also proven by the 166 sequences from Genbank database. The results suggested that these specific sites were useful for identifying the fake velvet antlers.

Figure 5 shows the cluster analysis of velvet antlers based on partial COI sequences. As shown, all the individuals of $R$. tarandus were obviously clustered together in one clade, so were 166 related sequences collected in Genbank database, which suggested that all the individuals of $R$. tarandus were completely discriminated correctly. The results proved that partial COI sequences showed the excellent identification power of velvet antlers with a correct identification rate of 


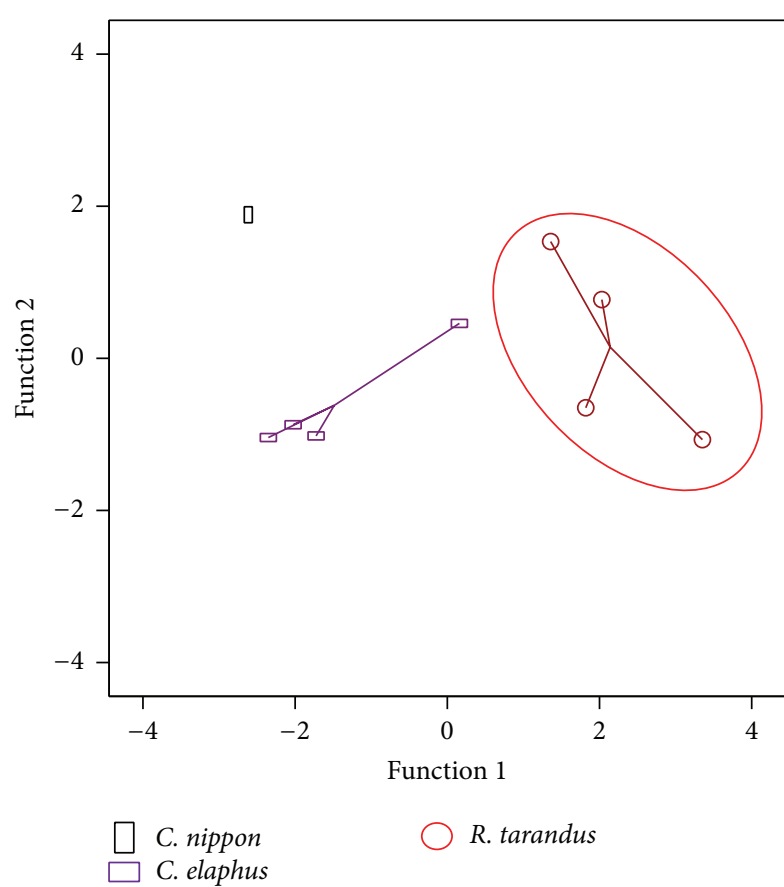

(a)

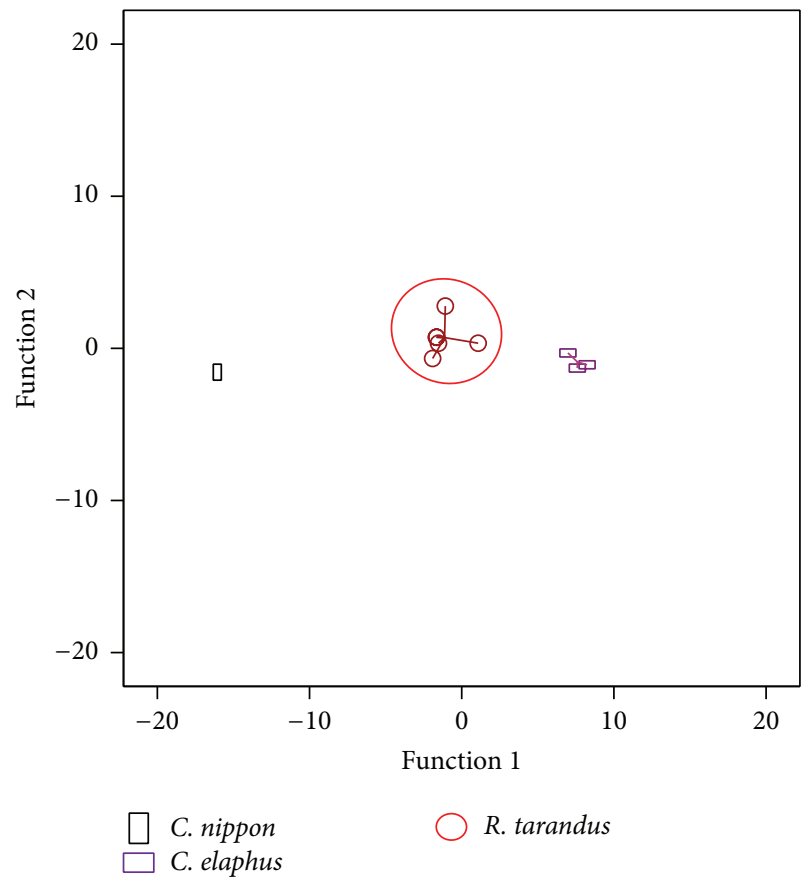

(c)

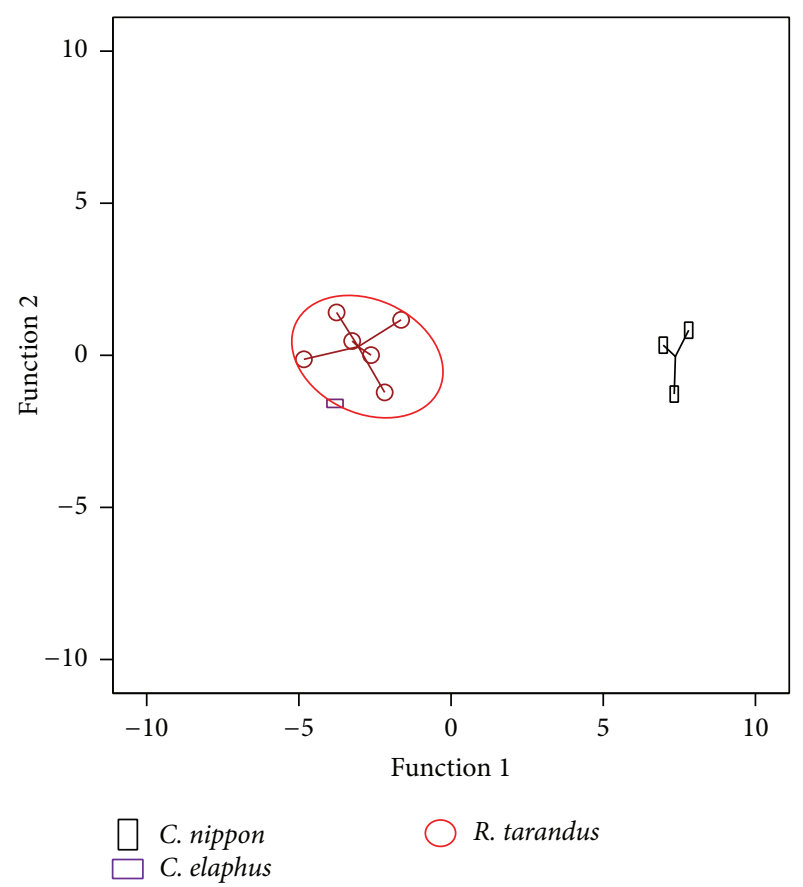

(b)

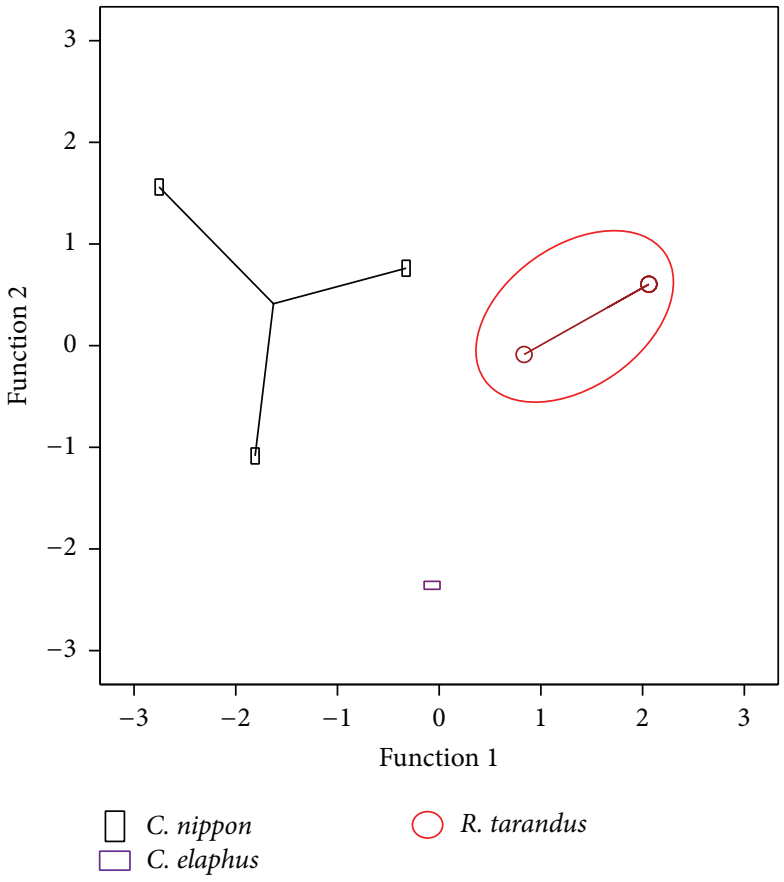

(d)

Figure 3: Data analysis of velvet antlers of different presentations based on LDA model. (a) Wax slices. (b) Powder slices. (c) Sand slices. (d) Bone slices.

$100 \%$. Thus, we determined DNA identification could be the good candidate for accurate identification.

DNA sequencing technology had been generally applied in the field of TCM in recent years $[4,15]$; it does have high accuracy, but it is also of high cost and is timeconsuming. So new methods in the future should be proposed to lower the cost and improve the efficiency. We thought that DNA specific amplification methods based on specific sites may come to our aid, such as allele-specific PCR [15] and amplification refractory mutation system [16]. These methods can detect specific DNA sites without sequencing, thus lowering the cost and improving the efficiency. And the better one, we believed, is loop-mediated isothermal amplification [17], which can rapidly detect samples in a few 


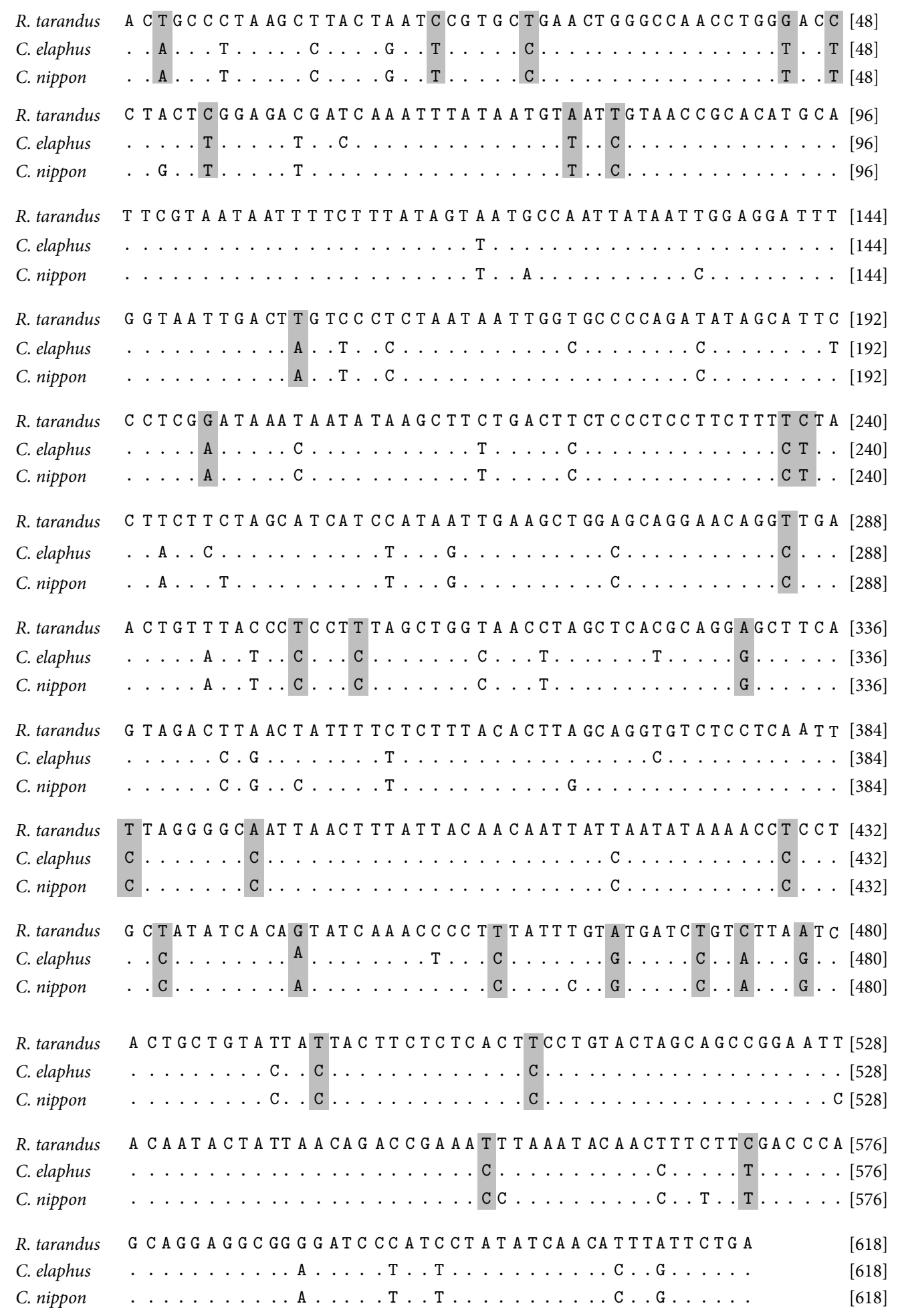

FIGURE 4: Arrangement of all specific sites of fake velvet antlers. minutes without PCR amplifier, and will play an important role in the further work. Another nut we have to be faced with is the DNA extraction of Chinese medicine. As we all know, lots of secondary metabolites are in Chinese medicines; development of novel DNA extraction and purification methods for Chinese medicines will be important in the future.
3.3. Application and Perspective of the Electronic Nose and DNA Identification Platform. It had been reported that the traditional authentication methods based on the morphology [12] and microscopic character $[11,18]$ had been firstly applied to discrimination of velvet antlers, but these methods are associated with high subjectivity. Development of chemical 


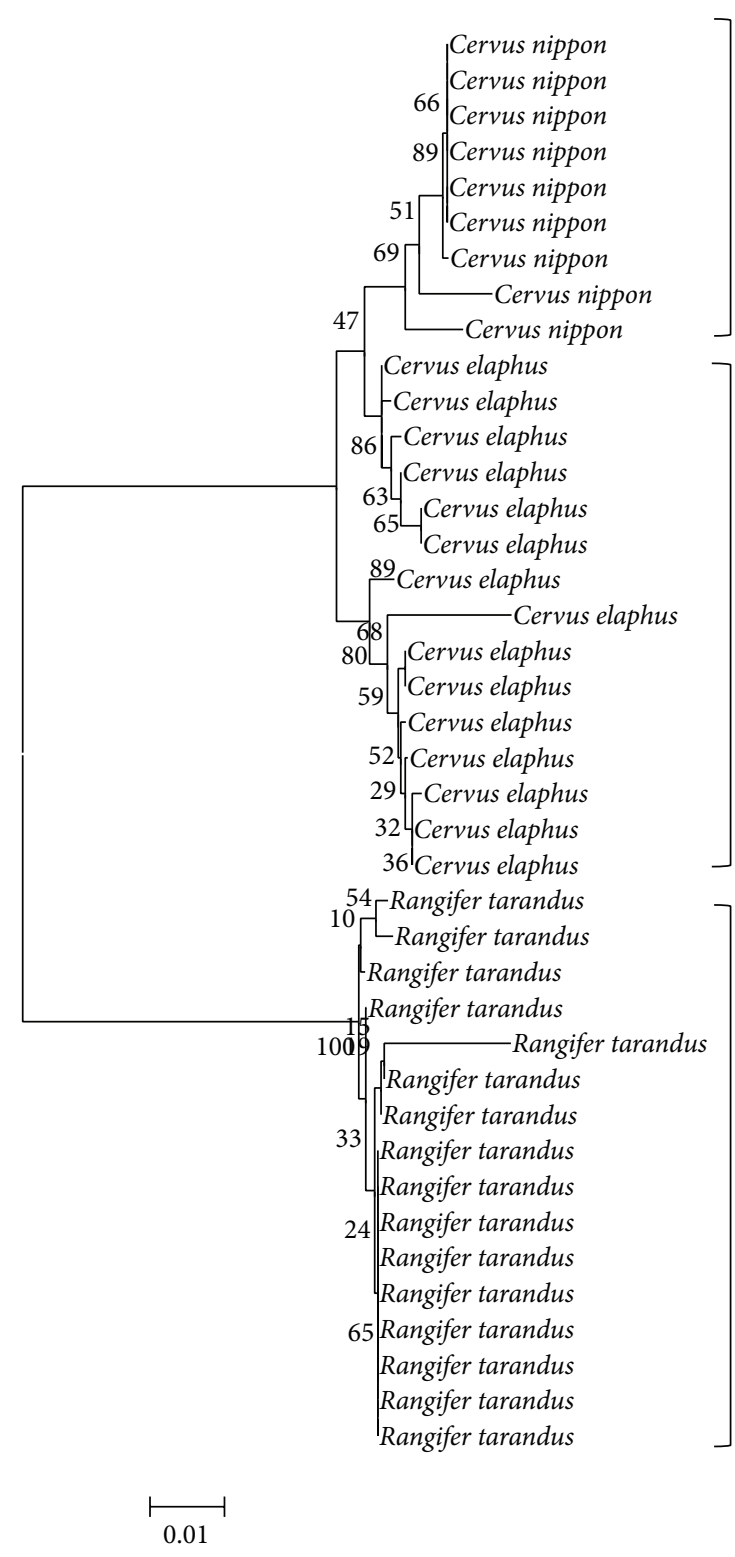

(a)

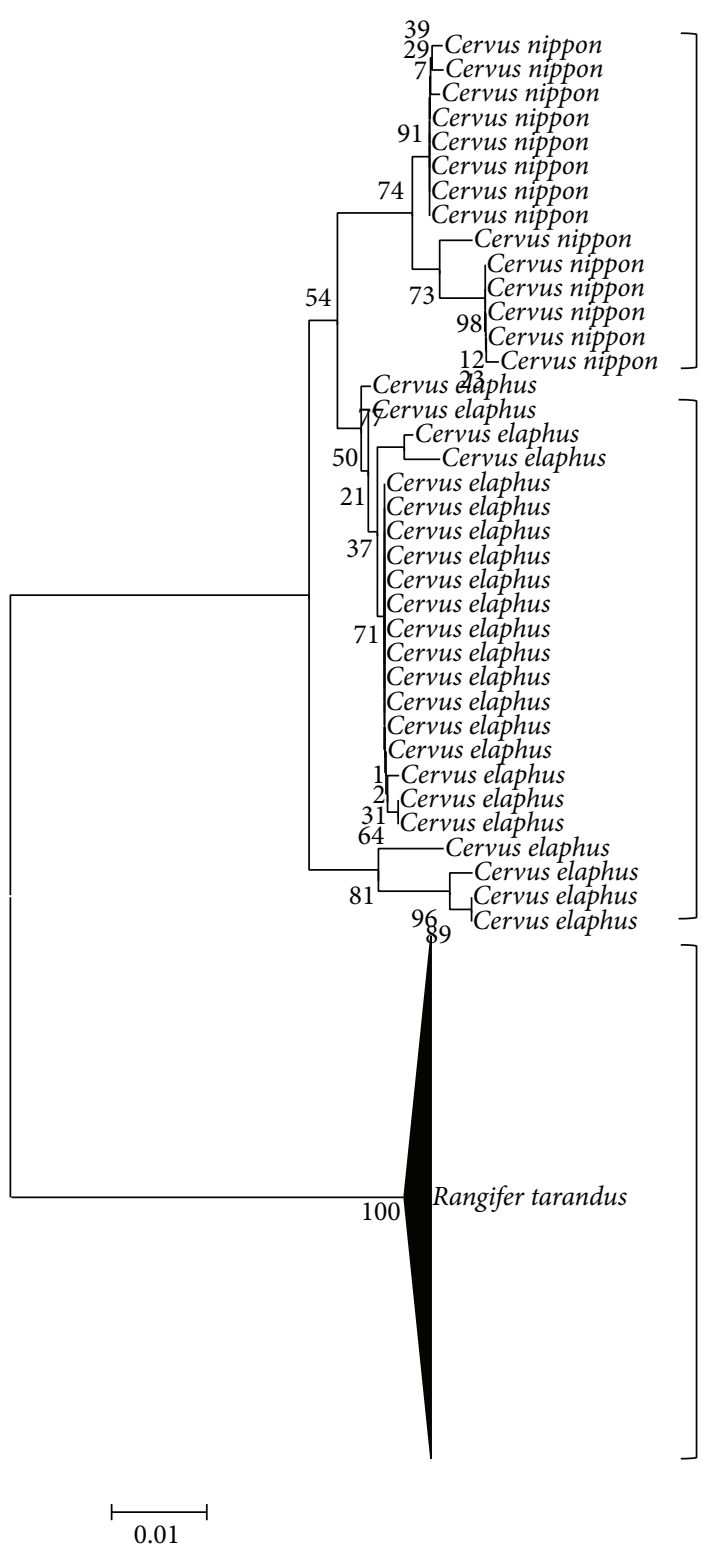

(b)

FIGURE 5: Cluster analysis of DNA sequences. (a) DNA sequences from materials of present study. (b) DNA sequences from Genbank database.

method based on high performance capillary electrophoresis (HPCE) [19] then had improved the objectivity and accuracy; nevertheless, it is always not satisfied in terms of practicality and identification power. In this study, electronic nose and DNA identification platform, integrating electronic nose and DNA sequencing technology, provides a more flexible and practical identification platform. Electronic nose is designed for rapid screening, while accurate identification based on DNA method is performed when electronic nose fails to accurately identify some complex samples, which does increase the efficiency of identification and vivify the use (Figure 1).

In fact, many traditional Chinese medicines are aromatic, such as herbal medicines from Umbelliferae and Labiatae, and electronic nose is exactly suitable for rapid identification of these materials. Meanwhile, DNA identification method, which is recognized as an accurate identification method universally, can make up for defects of electronic nose in case of unclear identification.

Electronic nose and DNA identification platform developed in this study will also be improved according to the vision mentioned above in our further study. We believe it will play an important role in the field of TCM.

\section{Conclusions}

In this study, rapid identification of different velvet antlers based on electronic nose showed the good identification power, while accurate identification based on DNA sequencing technology showed a correct identification rate of $100 \%$. 
The results further proved that electronic nose and DNA identification platform, covering the advantages of electronic nose and DNA sequencing technology, was effective for rapid and accurate identification and highly improved efficiency and decreased cost of identification. It is also believed that electronic nose and DNA identification platform will be of great significance to standardization, normalization, and medication safety of Chinese medicine.

\section{Abbreviations \\ TCM: Traditional Chinese medicine \\ HPCE: High performance capillary electrophoresis \\ LDA: Linear discriminant analysis \\ COI: Cytochrome oxidase subunit I.}

\section{Conflict of Interests}

The authors declare that they have no competing interests.

\section{Authors' Contribution}

Xueyong Wang and Chunsheng Liu conceived and designed the study. Guojie Xu and Caili Liao accomplished the whole experiment and wrote the paper. Xinyue Zhang, Xiaorui Fu, and Xiaolei Ren accounted for data analysis, collecting samples, and processing samples. All authors read and approved the final version of the paper.

\section{Acknowledgments}

The authors thank Huihui Duan of Beijing University of Chinese Medicine for supporting the study. This work was supported by Beijing Natural Science Foundation of China (7152094 and 7142101) and National Natural Science Foundation of China (81274011).

\section{References}

[1] K.-H. Kim, K.-S. Kim, B.-J. Choi et al., "Anti-bone resorption activity of deer antler aqua-acupunture, the pilose antler of Cervus koreanTEMMINCK var. mantchuricus Swinhoe (Nokyong) in adjuvant-induced arthritic rats," Journal of Ethnopharmacology, vol. 96, no. 3, pp. 497-506, 2005.

[2] K.-S. Kim, Y.-H. Choi, K.-H. Kim et al., "Protective and anti-arthritic effects of deer antler aqua-acupuncture (DAA), inhibiting dihydroorotate dehydrogenase, on phosphate ionsmediated chondrocyte apoptosis and rat collagen-induced arthritis," International Immunopharmacology, vol. 4, no. 7, pp. 963-973, 2004.

[3] J.-H. Lin, L.-X. Deng, Z.-Y. Wu, L. Chen, and L. Zhang, "Pilose antler polypeptides promote chondrocyte proliferation via the tyrosine kinase signaling pathway," Journal of Occupational Medicine and Toxicology, vol. 6, no. 1, article 27, 2011.

[4] D. Yan, J. Y. Luo, Y. M. Han et al., "Forensic DNA barcoding and bio-response studies of animal horn products used in traditional medicine," PLoS ONE, vol. 8, no. 2, Article ID e55854, 2013.
[5] X. Wang, C. Liu, R. Zhang, L. Huang, and G. Cui, "Establishment of allele-specific diagnostic PCR method for identification of zantlers," Zhongguo Zhongyao Zazhi, vol. 34, no. 23, pp. 30133016, 2009.

[6] R. Zhang, C.-S. Liu, L.-Q. Huang, X.-Y. Wang, G.-H. Cui, and L. Dong, "Study on the identification of Cornu Cervi Pantotrichum with DNA barcoding," Chinese Pharmaceutical Journal, vol. 46, no. 4, pp. 263-266, 2011.

[7] M. Natonek-Wioeniewska, E. Słota, and B. Kalisz, "Use of cytochrome b polymorphism for species identification of biological material derived from cattle, sheep, goats, roe deer and red deer," Folia Biologica, vol. 58, no. 1-2, pp. 47-50, 2010.

[8] Q. Wang, X. Zhang, H.-Y. Zhang et al., "Identification of 12 animal species meat by T-RFLP on the $12 \mathrm{~S}$ rRNA gene," Meat Science, vol. 85, no. 2, pp. 265-269, 2010.

[9] G. D. Haynes and E. K. Latch, "Identification of novel single nucleotide polymorphisms (SNPs) in deer (Odocoileus spp.) using the BovineSNP50 Beadchip," PLoS ONE, vol. 7, no. 5, Article ID e36536, 2012.

[10] Y. H. Kim, E. S. Kim, B. S. Ko et al., "A PCR-based assay for discriminating Cervus and Rangifer (Cervidae) antlers with mitochondrial DNA polymorphisms," Journal of Animal Science, vol. 90, no. 7, pp. 2075-2083, 2012.

[11] L. Liu and T.-G. Kang, "Microscopical identification and hierarchical cluster analysis of seven kinds of pilose antler velvet," Zhong Yao Cai, vol. 32, no. 3, pp. 345-347, 2009.

[12] D. Chen, Y. Guo, and W. Ren, "Character identification of 12 kinds of pilose antler medicinal materials," Zhong Yao Cai, vol. 22, no. 9, pp. 441-444, 1999.

[13] G. Xu, C. Liao, X. Ren et al., "Rapid assessment of quality of deer antler slices by using an electronic nose coupled with chemometric analysis," Brazilian Journal of Pharmacognosy, vol. 24, no. 6, pp. 716-721, 2014.

[14] K. Tamura, D. Peterson, N. Peterson, G. Stecher, M. Nei, and S. Kumar, "MEGA5: molecular evolutionary genetics analysis using maximum likelihood, evolutionary distance, and maximum parsimony methods," Molecular Biology and Evolution, vol. 28, no. 10, pp. 2731-2739, 2011.

[15] G. Xu, X. Wang, C. Liu et al., "Authentication of official Dahuang by sequencing and multiplex allele-specific PCR of a short maturase K gene," Genome, vol. 56, no. 2, pp. 109-113, 2013.

[16] X. Wang, G. Xu, C. Liu et al., "Development of deft amplification refractory mutation sequencing system (ARMSS) for discriminating Pilos antler based on a short cytochrome b (Cytb) gene," Mitochondrial DNA, 2014.

[17] K. Hsieh, B. S. Ferguson, M. Eisenstein, K. W. Plaxco, and H. T. Soh, "Integrated electrochemical microsystems for genetic detection of pathogens at the point of care," Accounts of Chemical Research, vol. 48, no. 4, pp. 911-920, 2015.

[18] D. J. Ye, "Microscopic identification of pilose antler and deerhorn," Zhong Yao Tong Bao, vol. 11, no. 12, pp. 17-19, 1986.

[19] Z. Yan, R. Y. Yuan, C. Y. Wang, Z. H. Zhang, L. P. Cai, and J. K. Wang, "Study on fingerprint of Corn cervi pantotrichum by HPCE," Food Science and Technology, vol. 34, no. 4, pp. 254-258, 2009. 


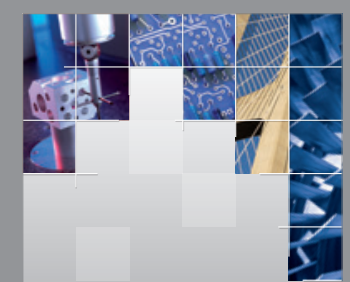

\section{Enfincering}
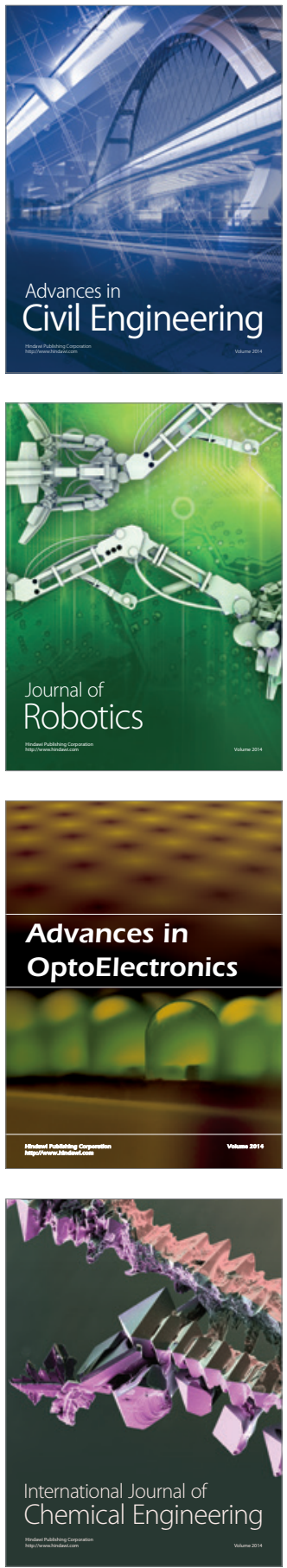

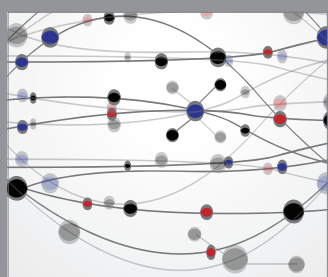

The Scientific World Journal

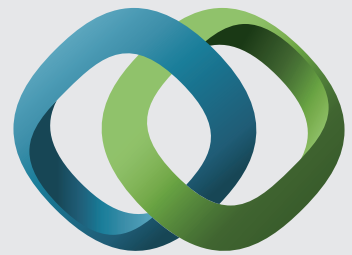

\section{Hindawi}

Submit your manuscripts at

http://www.hindawi.com
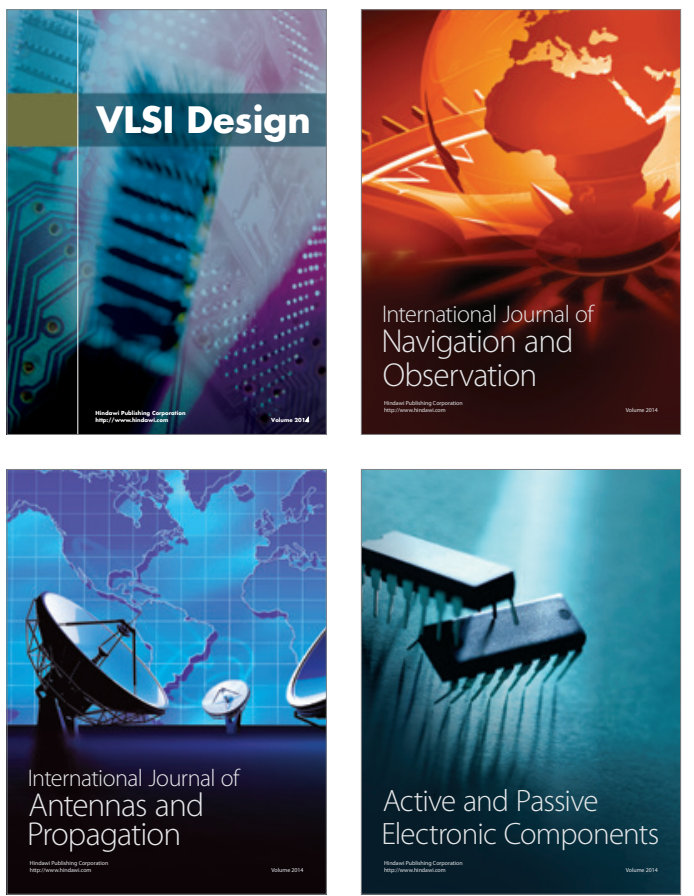
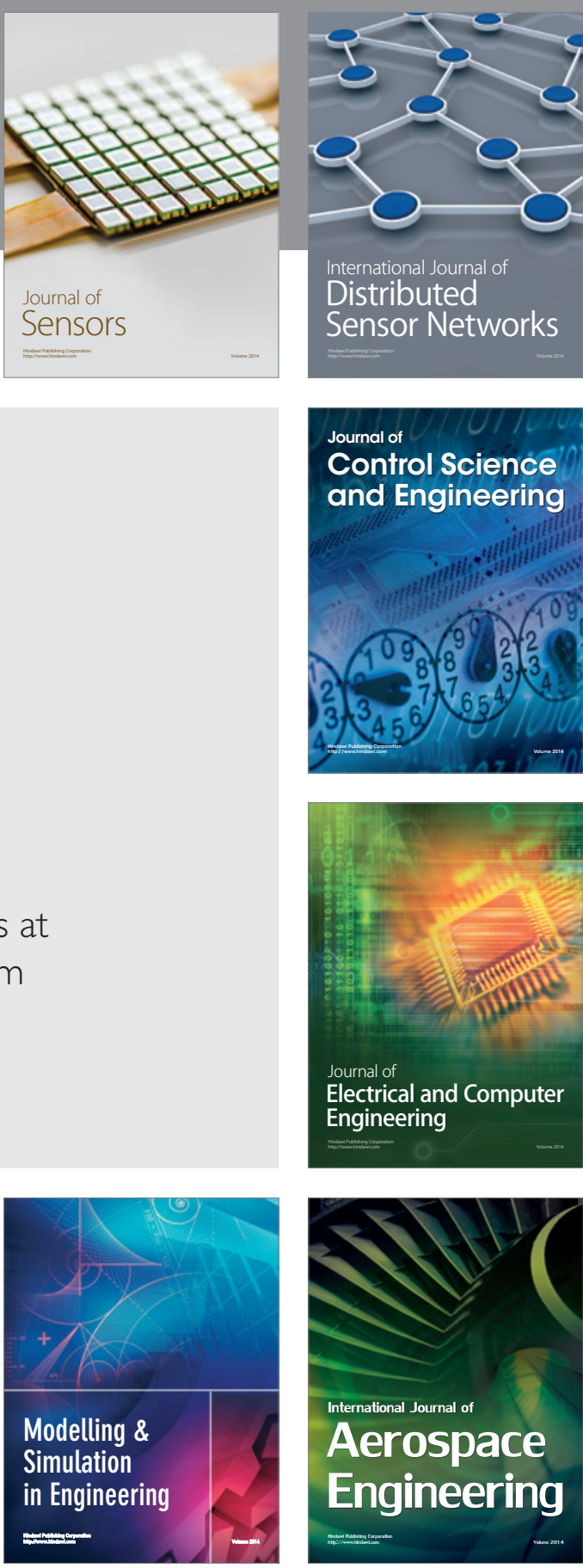

International Journal of

Distributed

Sensor Networks

Journal of

Control Science

and Engineering
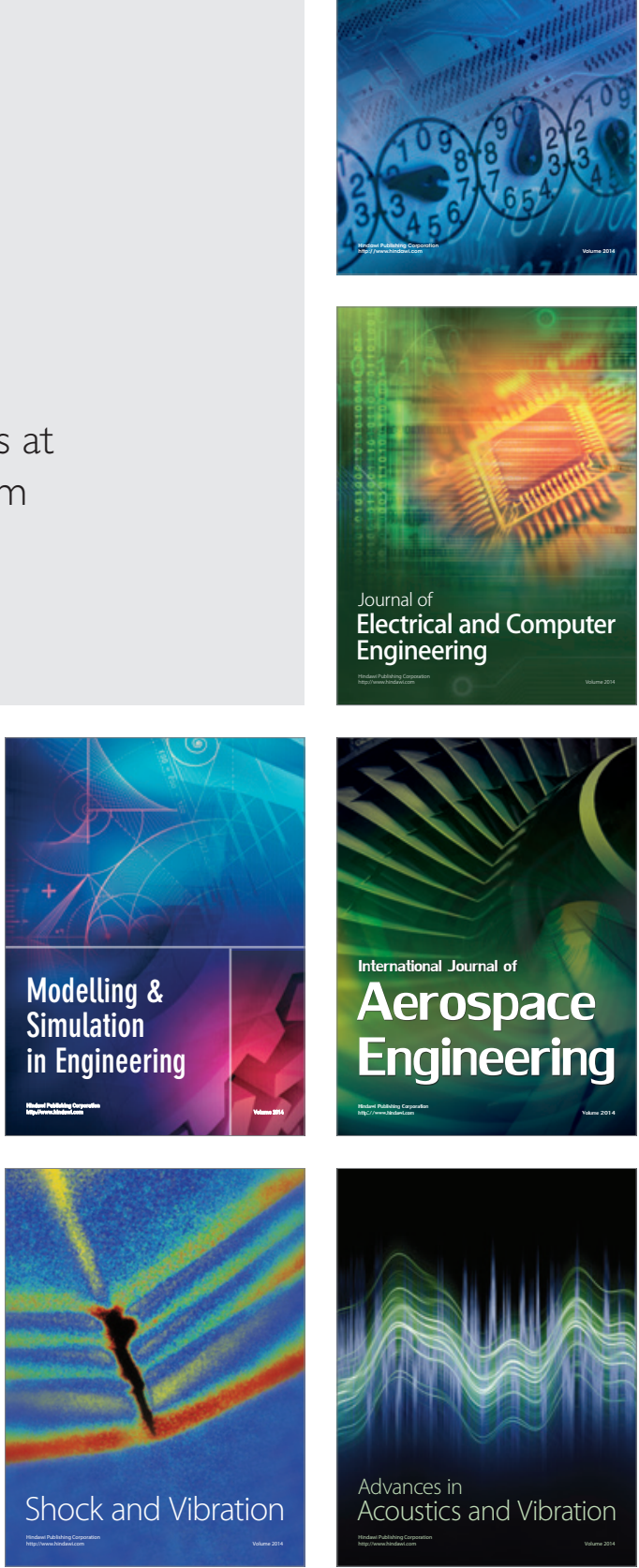